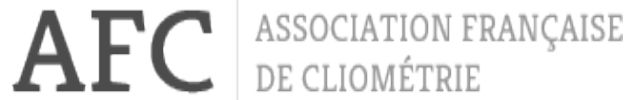

Nr. 11, 2006

Nonparametric Analysis of Financial Time

Series by the Kernel Methodology

Mohamed CHIKHI, Claude DIEBOLT 


\title{
Nonparametric Analysis of Financial Time Series by the Kernel Methodology
}

\author{
Mohamed CHIKHI ${ }^{1}$ \& Claude DIEBOLT ${ }^{2}$
}

\begin{abstract}
This paper aims to study, in the most recent historical time period, the efficiency of the Paris Stock Exchange market. We test its weak form while analysing the stock exchange returns series by nonparametric methods, using kernel methodology in particular. In doing so, our approach extends the traditional view treating the observed cyclical fluctuations on this market.
\end{abstract}

\section{Keywords}

Efficiency, random walk process, kernel methodology, functional autoregressive process, forecasting, cliometrics.

\section{JEL-classification}

C22, G14.

\footnotetext{
${ }^{1}$ Université de Ouargla \& LAMETA/CNRS, Université Montpellier I. Adresse: Faculté des Sciences Economiques, Espace Richter, Avenue de la Mer, C.S. 79606, 34960 Montpellier Cedex 2, France. Tel.: 33 (0)4.67.15.84.16, Fax.: 33 (0)4.67.15.84.67, E-mail: chikhi@lameta.univ-montp1.fr.

${ }^{2}$ CNRS, Université Louis Pasteur de Strasbourg \& Humboldt-Universität zu Berlin. Adresse: BETA/CNRS, Université Louis Pasteur de Strasbourg, Faculté des Sciences Economiques, 61 Avenue de la Forêt Noire, 67085 Strasbourg Cedex, France. Tel. (0)3.90.24.21.87, Fax. (0)3.90.24.20.71, E-mail: cdiebolt@cournot.u-strasbg.fr.
} 


\section{Introduction}

Economists have based work on the efficiency hypothesis since early 1950s. The approach stipulates that all the information relevant to the evaluation of financial assets is reflected instantly in prices. Such an approach therefore includes immediately the consequences of past events and precisely reflects anticipations expressed concerning future events. As a result, the price of a financial asset is at all times an unbiased estimate of its intrinsic value. It is therefore impossible to forecast its future variations as all the known or anticipated events have already been incorporated in its present value.

In fact, the efficient market hypothesis corresponds to the equilibrium theory applied to securities markets. The efficiency hypothesis assumes that agents are scattered. Participants are in active competition with the aim of making profits so none of them can be the sole influence on the prices that form on the market. Because of the active presence of a great number of market operators, the price differences observed in relation to the basic value will decrease. Furthermore, while the prices clearly reflect the information available, all the future events on which business profits depend are identified.

According to Jacquillat and Solnik (1997), the information-efficient market concept has extreme implications for portfolio management. Only investors who possess special information can hope to draw regular, abnormal returns.

In a general manner, efficiency tests are divided into three categories according to the information used in the forecasting attempt (Fama, 1991):

Weak efficiency: technical analysis is considered to be inoperative with low efficiency! Past prices cannot be used to forecast future prices. Only so-called historical information is included in the price. In other words, is it possible to make a profitable forecast when the information used in the forecasting attempt is limited to historical stock exchange data? Past prices play a central but not exclusive role in the information that can be used in this way. The data used here are the most easily available, the least subjective and the most widely disseminated. If it is possible not only to make forecasts using past prices but also to show a profit, the market is weak-form inefficient.

Semi-strong efficiency: here, all public information is added to the weak efficiency. This may consist of different press announcements or releases such as those concerning an increase in share capital for example. If it is possible to achieve gains greater than the expenses involved in the strategy, with management of a portfolio on the law of public information, the market is semi-strong form inefficient. 
Strong-form efficiency: in this case, the market as a whole has special information. There are no initiates and no returns greater than the market can be obtained. If the use of inside information is profitable then the market is said to be strong-form inefficient.

More generally, the efficiency theory of securities markets stipulates that the price observed reflects all the available information. This being said, all the empirical work conducted to date on efficiency theory is based on parametric methods. To the best of our knowledge, non-parametric methods have never been proposed in this context. Starting at this point, this article describes the use of a non-parametric study to examine the efficiency of the stock market in the case of France and more specifically the CAC40 share price index. We apply a test to the very recent historical period to see whether cyclical price fluctuations can be explained or not by a random market.

A short description of our method, that is to say a kernel estimator of the functional autoregressive process with non-parametric ARCH error, is provided in the next section. The efficiency hypothesis is tested on the French market, together with the unpredictable character of the data series studied.

\section{Nonparametric identification and estimation of a functional autoregressive models}

The nonparametric estimation of the univariate nonlinear autoregressive (NAR) process $\left\{Y_{t}\right\}_{t \geq 0}$ with $A R C H$ related errors is of the form:

$$
Y_{t}=f\left(X_{t}\right)+\sigma^{1 / 2}\left(X_{t}\right) \varepsilon_{t}
$$

with $X_{t}=\left(Y_{t-i_{1}}, \ldots \ldots . ., Y_{t-i_{k}}\right)^{\mathrm{T}}$ denoting the vector of length $\mathrm{k}$ of distinct lagged by $i_{1}<\ldots \ldots . .<i_{k}$ and $\varepsilon_{i} \sim \operatorname{IID}(0,1)$. Also note that the function $f($.$) represents the$ conditional expectation $E\left(Y_{t} \mid X_{t}\right)$. We assume that all lags $i_{1}, \ldots . ., i_{k}$ are needed for modelling $f($.$) but not necessarily \sigma($.$) .$

With regard to the process (1), we assume the following [see Doukhan (1994)]:

(H1) For an integer $M \geq i_{k}$, the vector process $X_{M, t}=\left(Y_{t-1}, \ldots . ., Y_{t-M}\right)^{\mathrm{T}}$ is strictly stationary $\beta$-mixing with $\beta(n) \leq c_{0} n^{-(2+\delta) / \delta}, \delta>0, c_{0}>0$ :

$$
\beta(n)=E \sup \left\{P\left(A \mid \mathfrak{R}_{M}^{k}\right)-P(A) \mid: A \in \mathfrak{R}_{n+k}^{\infty}\right\}
$$

Where $\mathfrak{R}_{t}^{t}$ is the $\sigma$-algebra by $X_{M, t}, X_{M, t+1}, \ldots \ldots, X_{M, t}$. 
(H2) The stationary distribution of the process $X_{M, t}$ has a density $\mu_{M}\left(x_{M}\right), x_{M} \in R^{M}$, which is continuous. If the Nadaraya-Watson estimator is used, $\mu_{M}($.$) has to be continuously differentiable.$

(H3) The function $f($.$) is twice continuously differentiable while \sigma($.$) is$ continuous and positive on the support of $f($.$) .$

(H4) The $\left\{\varepsilon_{t}\right\}_{t \geq i_{k}}$ have a finite fourth moment.

There are two popular, related nonparametric estimation techniques for estimating the conditional mean function and the volatility function for which the asymptotic properties have been derived recently: the Nadaraya-Watson estimator $\hat{f}_{1}(x)$ and the local linear estimator $\hat{f}_{2}(x)$.

Denote:

$$
X_{t}=\left(Y_{t-i_{1}}, Y_{t-i_{2}}, \ldots ., Y_{t-i_{k}}\right)^{\mathrm{T}}=\left(X_{t_{1}}, X_{t_{2}}, \ldots . ., X_{t_{k}}\right)^{\mathrm{T}}
$$

And

$$
Y=\left(Y_{i_{k}}, Y_{i_{k+1}}, \ldots . ., Y_{n}\right)^{\mathrm{T}}
$$

For all $x \in R^{k}$, the estimators can be written as:

$$
\begin{gathered}
\hat{f}_{1}(x)=\left(Z_{1}^{T} W Z_{1}\right)^{-1} Z_{1}^{T} W Z \\
\hat{f}_{2}(x)=e^{T}\left(Z_{2}^{T} W Z_{2}\right)^{-1} Z_{2}^{T} W Z \\
Z_{1}=(1, \ldots . ., 1)_{1 \times\left(n-i_{k}+1\right)}^{T}
\end{gathered}
$$

In which:

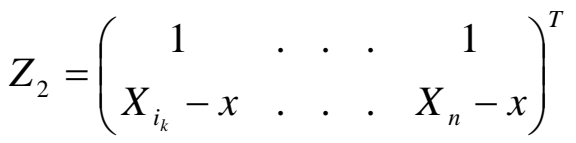

$$
\begin{aligned}
& e=\left(\begin{array}{ll}
1 & 0_{1 \times k}
\end{array}\right)^{T} \\
& W=\operatorname{diag}\left\{\frac{1}{n^{\prime}} K_{h}\left(X_{i}-x\right)\right\}_{i=i_{k}}^{n}
\end{aligned}
$$

With: $n^{\prime}=n-i_{k}+1$

(H5) $K: R \rightarrow R$ is a symmetric probability density (kernel) and $h=h_{n}$ is a positive number (bandwidth) with $h \rightarrow 0, n h^{k} \rightarrow \infty$ as $n \rightarrow \infty$.

We denote $\|K\|_{2}^{2}=\int K^{2}(u) d u, \sigma_{K}^{2}=\int K(u) u^{2} d u$. For all $x \in R^{k}$, we write:

$$
K_{h}(x)=\frac{1}{h^{k}} \prod_{j=1}^{k} K\left(\frac{x_{j}}{h}\right)
$$

The Nadaraya-Watson estimator is just a special case of the local linear estimator since it only locally estimates a function that is constant. The asymptotic convergence 
and asymptotic normality of the Nadaraya-Watson estimator for the nonlinear $A R$ ARCH model was derived first by Masry and Tjostheim (1995). However, Härdle, Tsybakov and Yang (1996) derived the asymptotic bias variance and normality for nonlinear vector autoregressive models with a conditional heteroscedasticity for the univariate case:

$$
\begin{aligned}
& \sqrt{n^{\prime} h^{k}}\left\{\hat{f}_{1}(x)-f(x)-h^{2}\left(b_{c}(x)+b_{t}(x)\right)\right\} \stackrel{D}{\longrightarrow} N(0, V(x)) \\
& \sqrt{n^{\prime} h^{k}}\left\{\hat{f}_{2}(x)-f(x)-h^{2} b_{t}(x)\right\} \stackrel{D}{\longrightarrow} N(0, V(x))
\end{aligned}
$$

As $n \rightarrow \infty$, with asymptotic bias:

$$
\begin{gathered}
b_{c}(x)=\frac{\sigma_{K}^{2}}{2}\left\{\frac{2 \nabla f(x)^{\mathrm{T}} \nabla \mu(x)}{\mu(x)}\right\} \\
b_{l}(x)=\frac{\sigma_{K}^{2}}{2} \operatorname{Tr}\left[\nabla^{2} f(x)\right]
\end{gathered}
$$

And asymptotic variance:

$$
V(x)=\frac{\sigma(x)}{\mu(x)}\|K\|_{2}^{2 k}
$$

Where $\|K\|_{2}^{2}=\int K^{2}\left(x_{j}\right) d x_{j}$ and $\sigma_{K}^{2}=\int x_{j}^{2} K\left(x_{j}\right) d x_{j}$

The asymptotic bias of the Nadaraya-Watson estimator is more complicated than the asymptotic bias of the local linear estimator. In addition to the sum of the direct second derivatives of the function $f(x)$ with respect to all the regressors that is common to both expressions it also depends on the conditional density function $\mu(x)$ and its first derivatives as well as the first derivatives of the function $f(x)$. This implies for example that as long as $D G P$ is linear, the local linear estimator has no bias while for the Nadaraya-Watson estimator this is only true if the DGP is constant. In contrast, both estimators exhibit the same asymptotic variance that depends on the conditional volatility function $\sigma(x)$ and the density of $x$. Similar estimators and their asymptotic behaviour have been derived for estimating $\sigma(x)$ [see Masry and Tjostheim (1994), Härdle, Tsybakov and Yang (1996)]. Such estimates can also be used to construct confidence intervals for $\hat{f}_{i}(x), i=1,2$. It should also be pointed out that the convergence rate of both estimators depends on the dimension of the regressor matrix.

A bandwidth and a vector of lags must always be specified to apply the local linear estimator to estimate the $N A R-A R C H$ model. Nonparametric model selection is concerned with methods for choosing these numbers. While cross validation has long been used for estimating optimal bandwidth, it is only recently that some of its 
asymptotic properties have been investigated for lag selection [Vieu (1994) and Yao and Tong (1994)]. An alternative approach that extends the linear Final Prediction Error (FPE) to nonparametric estimators was pursed by Tjosthein and Auestad (1994) on the basis of the Nadaraya-Watson estimator. However, they did not prove the consistency of their approach. This was done by Tschernig and Yang (1996) who allowed for both the local constant as well as the local linear estimator. In addition, they derived an optimal bandwidth estimate and derived the rates of the asymptotic probabilities of over- and under-fitting. Tschernig and Yang (1998) show that the asymptotic FPE gives a better rate of convergence than the cross-validation method. The other authors used only the Nadaraya-Watson estimator for their lag selection procedures. However, the Nadaraya-Watson estimator has a poor bias rate if the density of the lagged variable is not sufficiently smooth, especially with nonlinear processes. In contrast, the local linear estimator only needs continuity of the density to have an optimal convergence rate [see, for example, Härdle and Al (1998)].

Let $\left\{\tilde{Y}_{t}\right\}$ be another series with exactly the same distribution as $\left\{Y_{t}\right\}$ but independent of $\left\{Y_{t}\right\}$. The Final Prediction Error (FPE) of an estimate $\hat{f}$ of $f$ is defined as the following functional:

$$
\operatorname{FPE}(\hat{f})=\lim E\left[\left\{\tilde{Y}_{t}-\hat{f}\left(\tilde{X}_{t}\right)\right\}^{2} w\left(\tilde{X}_{M, t}\right)\right]
$$

Where the expectation is taken over all the variables $Y_{0}, Y_{1}, \ldots \ldots, Y_{n}, \tilde{Y}_{0}, \tilde{Y}_{1}, \ldots \ldots, \tilde{Y}_{n}$. For the weight function $w: R^{M} \rightarrow R$ we assume:

(H6) The support of $w($.$) is compact with nonempty interior. The function w($.$) is$ continuous, non-negative, and $\mu\left(x_{M}\right)>0$ for $x_{M} \in \operatorname{supp}(w)$.

If the process $\left\{Y_{t}\right\}$ is a stationary linear AR process, $\hat{f}$ is a linear regressor, the FPE becomes the usual linear FPE introduced by Akaike (1971), if the process $\left\{Y_{t}\right\}$ is a stationary nonlinear $A R$ process and $\hat{f}$ a nonparametric estimator, we have the nonparametric FPE. For these estimators, it can be shown that (7) asymptotically consists of three parts:

$$
\operatorname{FPE}_{a}(h)=\operatorname{AFPE}_{a}(h)+o\left(h^{4}+\frac{1}{n h^{k}}\right)
$$

Where:

$$
\operatorname{AFPE}_{a}(h)=A+b(h) B+c(h) C_{a}
$$


With:

$$
\begin{gathered}
A=\int \sigma(x) w\left(x_{M}\right) \mu\left(x_{M}\right) d x_{M}, B=\int \sigma(x) w\left(x_{M}\right) \mu\left(x_{M}\right) / \mu(x) d x_{M}, \\
C_{a}=\int r_{a}^{2}(x) w\left(x_{M}\right) \mu\left(x_{M}\right) d x_{M} \\
r_{1}(x)=\operatorname{Tr}\left\{\nabla^{2} f(x)\right\}+2 \nabla^{T} \mu(x) \nabla f(x) / \mu(x) \\
r_{2}(x)=\operatorname{Tr}\left\{\nabla^{2} f(x)\right\}
\end{gathered}
$$

Where:

$$
b(n)=\|K\|_{2}^{2 k} n^{\prime-1} h^{-k}, c(h)=\sigma_{K}^{4} h^{4} / 4
$$

The index $a=1,2$ indicates the local constant and local linear estimator respectively. The first part of the formula (8) describes the expected white noise variance of the identical processes $\left\{Y_{t}\right\}$ and $\left\{\tilde{Y}_{t}\right\}$. The second term $B$ denotes the expected value of the variance of the nonparametric estimator $\hat{f}_{a}$; while the third term captures its expected estimation bias. Both estimators only differ with respect to their estimation bias; it should not be surprising that both asymptotic FPE only differ in the bias term. It is worth noting that all expected values are independent of the bandwidth $h$ and are solely determined by the properties of the DGP. Furthermore, as more data become available both the expected variance and bias term become negligible and the AFPE $(h)$ approaches its asymptotic minimum $E(\sigma(x))$ since then $h \rightarrow 0$ and $n h^{k} \rightarrow \infty$ for $n \rightarrow \infty$. However, for a given sample size $n$, they make it possible to determine an optimal bandwidth $h_{a, o p t}$ by minimising the sum of the second and the third term:

$$
h_{a, o p t}=\left\{k\|K\|_{2}^{2 k} B n^{\prime-1} C_{a}^{-1} \sigma_{K}^{-4}\right\}^{1 /(k+4)}
$$

and the minimal AFPE criterions given by:

$$
A F P E_{a, o p t}=A+\left(k^{-k /(k+4)}+\frac{1}{4} k^{4 /(k+4)}\right)\left\{\mid K \|_{2}^{8 k} B^{4} n^{\prime-1} C_{a}^{k} \sigma_{K}^{4 k}\right\}^{1 /(k+4)}
$$

Equation (9) is necessary to obtain the estimate of $h_{a, o p t}$ and expression (8) contains the unknown quantities $A, B$ and $C$. The following estimates of $A$ and $B$ are required for estimating the asymptotic FPE:

$$
\begin{gathered}
\hat{A}_{a}=n^{\prime-1} \sum_{i=i_{k}}^{n}\left\{Y_{i}-\hat{f}_{a}\left(X_{i}\right)\right\}^{2} w\left(X_{M, i}\right) \\
\hat{B}_{a}=n^{\prime-1} \sum_{i=i_{k}}^{n}\left\{Y_{i}-\hat{f}_{a}\left(X_{i}\right)\right\}^{2} w\left(X_{M, i}\right) / \hat{\mu}\left(X_{i}\right)
\end{gathered}
$$


The estimators $\hat{f}_{a}$ use bandwidths of the same order $n^{-1 /(k+4)}$ as the optimal $h_{a, \text { opt }}$ and $\hat{\mu}\left(X_{i}\right)$ is a kernel estimator of the density. As $A$ is the dominant term in the AFPE expression, we look at the asymptotic of $\hat{A}_{a}$, which estimates the mean squared error. Under assumptions (H1)-(H6), for $a=1,2$ as $n \rightarrow \infty$,

$$
\begin{aligned}
\hat{A}_{a}=A+\left\{\|K\|_{2}^{2 k}-2 K(0)^{k}\right\} n^{-1} h^{-k} B & +C_{a} \sigma_{K}^{4} h^{4} / 4+ \\
& o\left\{h^{4}+n^{\prime-1} h^{-k}\right\}+O_{P}\left\{n^{\prime-1 / 2}\right\}
\end{aligned}
$$

Note that with bandwidth $h$ of the form $\mathrm{cn}^{-1 /(k+4)}$, the nonparametric estimate $\hat{A}_{a}$ converges to $A$ at the parametric $\sqrt{n}$ if $k \leq 4$. In which case the second and third term will be $\mathrm{O}\left\{n^{1-1 / 2}\right\}$.

The nonparametric estimate of the asymptotic FPE is:

$$
A F P E_{a}=\hat{A}_{a}+2 K(0)^{k} n^{\prime-1} h_{a, o p t}^{-k} \hat{B}_{a}
$$

$\hat{A}_{a}$ is evaluated using the optimal bandwidth $h_{a, \text { opt }}$, while $\hat{B}_{a}$ using any bandwidth of order $n^{1-1 /(k+4)}$.

Tschernig and Yang (1996) show that these nonparametric FPE estimators are consistent under standard conditions used in nonparametric time series analysis. However, as they prove there is a larger asymptotic probability to over-fit than to underfit the correct model, where over-fitting is meant to always include all correct lags plus some irrelevant lags. They therefore suggest a correction factor by which the $A F P E_{a}$ :

$$
\operatorname{CAFPE}_{a}=\operatorname{AFPE}_{a}\left(1+\frac{k}{n^{\frac{1}{k+4}}}\right)
$$

where the correction depends on the number of lags $k$ and the number of observations $n$. Now the selection rule is to choose the subset $\left\{\hat{i}_{1}, \ldots \ldots ., \hat{i}_{\hat{k}}\right\}$ under assumptions (H1)-(H6), such as [cf. Tschernig and Yang (1998), p.7]:

$$
P\left\{\hat{k}=k, \hat{i}_{l}=i, l=1, \ldots \ldots, k\right\} \underset{n \rightarrow \infty}{\longrightarrow} 1
$$

The nonparametric predictors of the horizon $h$ for the $N A R-A R C H$ process that are defined by:

$$
\begin{gathered}
f_{h}(x)=E\left(Y_{t+h-1} \mid X_{t-1}=x\right) \\
Y_{t+h-1}=f_{h}\left(X_{t-1}\right)+\sigma_{h}\left(X_{t-1}\right) \cdot \varepsilon_{t, h}
\end{gathered}
$$

Where:

$$
\sigma_{h}^{2}(x)=\operatorname{var}\left(Y_{t+h-1} \mid X_{t-1}=x\right)
$$


The local linear estimator of the prediction function $f_{h}(x)$ is written:

$$
\begin{gathered}
\hat{f}_{h}(x)=e^{T}\left(Z_{2 h}{ }^{T} W_{h} Z_{2 h}\right)^{-1} Z_{2 h}{ }^{T} W_{h} Z_{h} \\
\hat{\sigma}_{h}(x)=\left\{e^{T}\left(Z_{2 h}{ }^{T} W_{h} Z_{2 h}\right)^{-1} Z_{2 h}{ }^{T} W_{h} Z_{h}^{2}-\hat{f}_{h}{ }^{2}(x)\right\}^{1 / 2}
\end{gathered}
$$

Where:

$$
\begin{aligned}
& e^{T}=\left(\begin{array}{ll}
1 & 0_{1 \times k}
\end{array}\right)^{T},
\end{aligned}
$$

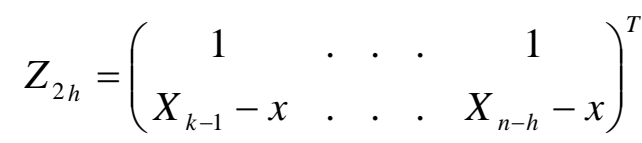

$$
\begin{aligned}
& W_{h}=\operatorname{diag}\left\{K_{h}\left(X_{i-1}-x\right) / n\right\}_{i=k}^{n-h+1}
\end{aligned}
$$

And:

$$
Y_{h}=\left(Y_{k+h-1} \cdot \cdot \cdot Y_{n}\right)^{T}
$$

\section{Econometric analysis}

The efficiency of the French Stock Market was examined. The data used here are the daily CAC40 index series for the period 09/07/1987-05/28/1999, with 3060 observations. We retain a daily frequency in the data insofar as if the efficiency hypothesis is rejected on the daily data, it should also be rejected on the weekly or the monthly data.

The implementation of the independence tests and the nonparametric analysis tools requires that the series be stationary. The Augmented Dickey-Fuller unit-root test shows that one unit root exists in the CAC40 series (see Table 1). The Phillips-Perron and Breitung's nonparametric unit root tests are applied in order to confirm these results. These two tests are particularly important within the framework of the financial series because they are robust with the heteroscedasticity. They show also that the presence of a unit root in the price series is confirmed (see Table 2). So, this series is logdifferenced to give the Stock Exchange returns in Figure 2.

As is shown in Table 3, there is strong evidence that the null hypothesis of normality is rejected for the Stock Exchange returns. Leptokurtosis in the data is revealed by a high Kurtosis coefficient of 9.21 and a Jarque-Bera statistic of 5017.6. The nonparametric kernel density estimator confirms these results. Figure 3 shows the asymmetry and leptokurtic character of the distribution. This asymmetry may be caused by the presence of nonlinearities in this series. Moreover, the scatter diagram (see Figure 4) confirms these results where the obtained form is irregular ellipsoid and the distribution is typically non-Gaussian. This nonlinearity can testify, for example, to the presence of an $A R C H$ effect, frequently encountered in financial time series. Table 4 presents the results of White and Breusch-Pagan heteroscedasticity tests. They show 
that the null hypothesis of homoscedasticity is rejected at $5 \%$ and so this returns series is heteroscedastic. It is noted thus that the presence of a conditional heteroscedasticityindicating that the returns are not iid- does not indicate market inefficiency.

As can be seen in Tables 5 and 6, we note that the random walk hypothesis is clearly rejected. The nonparametric Mizrach and BDS statistics, which test for the presence of a linear or nonlinear dependence, are greater than the critical value at $5 \%$. Therefore, the stock exchange returns process is mixing and predictable and this market is not weakly efficient. These tests generally show the presence of a significant autocorrelations different to zero in the short term. It is impossible to exploit these autocorrelations to establish a speculation rule leading to abnormal benefits. Consequently, we judge that these results do not enable us to conclude clearly in favour of inefficiency markets. Various authors test this hypothesis while working on longer horizons. The nonparametric Mizrach and BDS tests led us to reject the null iid hypothesis but they cannot detect the presence of a long term dependence structure. For this reason, we retain in this application to the Stock Exchange returns series various values for the ordinates of the periodogram in order to frame the squared root number of observations. We examine the stability of the estimators when the number of the periodogram varies. As is seen in Table 7, there is strong evidence that the CAC40 returns series exhibits short memory. The statistic values are smaller than the critical value at 5\%. Nevertheless, the Stock Exchange returns are predictable in the short term. The presence of short-term dynamics indicates that the agents cannot anticipate their returns at sufficiently long horizons to allow them to 'beat the market'. The nonlinearity hypothesis of such processes seems acceptable. Consequently, the modelling of our series turns towards a nonlinear process. As it is very difficult to identify a nonlinear parametric process, we use the nonparametric approach based on the local linear estimator.

Any nonparametric model fitting requires the selection of the relevant lags and bandwidth. Optimally this is done within the same model class as the estimation itself. For that, we use the nonparametric CAFPE.

In all cases, the maximum number of lags is restricted to be five and the largest lag to be considered is height. This amounts to checking $1+\sum_{j=1}^{5}\left(\begin{array}{l}8 \\ j\end{array}\right)$ different lag combinations for a full search procedure. Tjostheim and Auestad (1994) suggest adding lags as long as they reduce the selection criterion. However, their key idea is to choose the lags with respect to their contribution to this reduction. To be more precise, in the first step the most important lag $\hat{i}_{1}$ is determined by selecting $i_{1}$ such that the selection 
criterion is minimum among all one-lag models. If the criterion value is comparable to the variance of the data, no significant lag is included. Otherwise $\hat{i}_{2}$ is found in the next step by keeping $\hat{i_{1}}$, fixed and choosing $i_{2}$ in such a way that the selection criterion is minimised for both lag models. Again, $\hat{i}_{2}$ is only included and the procedure continued if the two lag criterion value is smaller than the own lag criterion value. A similar method is then used to determine $\hat{i}_{3}, \ldots . ., \hat{i}_{d}$ where $d \leq 5$.

A bandwidth must be specified for each computation of the CAFPE 'Corrected Asymptotic Final Prediction Error'. This can be done either by estimating the optimal bandwidth $h_{a, o p t}$ or by conducting a grid search. The latter is justified by the existence of the optimal bandwidth. For the local constant model, estimation of the expected bias term would require estimation of the first derivatives of the density in $b_{c}\left(X_{t}\right)$ (4). As a result, the optimum bandwidth (9) is only estimated for the local linear model. The direct second derivatives needed for estimating $E\left(b_{t}\left(X_{t}\right)\right)$ can be estimated using a local quadratic estimator [cf. Tschernig and Yang (1996)] with bandwidth:

$$
h=2 \operatorname{var}\left(Z_{t}\right)\left(\frac{4}{k+10}\right)^{\frac{1}{k+12}} n^{-\frac{1}{k+12}}
$$

To compute the residuals and the density that are used to estimate the expected variance of $\hat{f}_{a}$, we use a Silverman's bandwidth [see Silverman (1986), eq. (4.14), p. 86]:

$$
h_{S}=\operatorname{var}\left(Z_{t}\right)\left(\frac{4}{k+2}\right)^{\frac{1}{k+4}} n^{-\frac{1}{k+4}}
$$

In both cases, additionally a leave-one-out technique is employed.

Moreover, the grid search procedure for determining the optimal bandwidth is used foe all CAFPE $E_{a}, a=1,2$ where the grid covers the interval $\left[0,2 h_{s}, 2 h_{s}\right]$ in 24 steps.

In order to estimate the Stock Exchange returns series, we use the Gaussian kernel. The weight function $w($.$) is specified by:$

$$
w\left(X_{t}\right)=\left\{\begin{array}{l}
1 \\
0
\end{array}\right\} \text { if }\left\{\begin{array}{l}
\hat{\mu}\left(X_{t}\right) \geq c \\
\hat{\mu}\left(X_{t}\right)<c
\end{array}\right.
$$

where the constant $c$ is determined such that $n$ " observations are excluded and $n$ " denotes the next larger integer of $0,05 n$ '. 
The results of the identification of the CAC40 index returns process are shown in Table 8. It also contains the lags chosen, the values of the criterion and the estimated optimum window. When using this non-parametric model, we noticed that the residuals (Fig. 5) are not characterised by a Gaussian distribution (Table 9); we also noted the leptokurtic nature of the residuals (Fig. 6). This asymmetry may indicate the presence of non-linear features in the residuals. The recurrence histogram (Fig. 7) confirms these results in which the residuals of the $N A R$ model are not white noise showing the existence of periodicity and non-linearity. However, the results of Mizrach's nonparametric test applied to the residuals displays a dependence structure (Table 10). These residuals can be modelled using the $A R C H$ models as the presence of an $A R C H$ effect is confirmed by the result of the $A R C H L M$ test on the residuals $\left(n R^{2}=7.698>\chi^{2}(1)\right)$. In general, financial series display variable volatility and asymmetry phenomena that cannot be allowed for in conditional expectation modelling. Description of the phenomenon requires the estimating of the volatility of returns using the non-parametric approach based on the kernel method.

The results of the estimation of the volatility of stock exchange returns are shown in Table 11. It contains the lags chosen, the value of the criterion and the optimum window. It is noted that the standardised residuals do not display any form of dependence (Table 12) and no conditional heteroscedasticity as $3.8179<\chi^{2}(1)$.

But, finally, is the stock market in France weak-form efficient? Comparison of the forecasting performances of non-parametric predictors with forecasts drawn from a simple random model was performed using four criteria: relative mean absolute error (RMAE), mean quadratic error (MQE), absolute mean error (AME) and residual standard deviation (RSD):

$$
\begin{gathered}
\text { MRAE }=H^{-1} \sum_{h=1}^{H} \frac{\left|\hat{Y}_{n-H+h}-Y_{n-H+h}\right|}{\left|Y_{n-H+h}\right|} \times 100 \\
\text { MAE }=H^{-1} \sum_{h=1}^{H}\left|\hat{Y}_{n-H+h}-Y_{n-H+h}\right| \\
\text { QME }=H^{-1} \sum_{h=1}^{H}\left(\hat{Y}_{n-H+h}-Y_{n-H+h}\right)^{2} \\
R S E=\left\{\sum_{h=1}^{H} H^{-1}\left[\hat{Y}_{n-H+h}-Y_{n-H+h}\right]^{2}-\left[\sum_{h=1}^{H} H^{-1}\left(\hat{Y}_{n-H+h}-Y_{n-H+h}\right)\right]^{2}\right\}^{1 / 2}
\end{gathered}
$$

Where:

$h$ is the horizon of prediction,

$H$ represents the sample for out-of sample prediction, 
The non-parametric model NAR-ARCH was re-estimated until 8 May 1999 and the 20 subsequent date sets were forecast. Forecasts are dynamic for all date horizons. In this study, the predictive qualities of the process are compared with the random operation model, whereas returns cannot be forecast from past values in the same series; this is supported by the market efficiency theory.

With regard to Table 13, it is noted that the forecasts provided by the non-parametric method are better than those resulting from a random operation model. We consider that this has a direct impact on the weak-form market efficiency. With non-parametric modelling, the series of CAC40 figures therefore seems to be forecastable in the short term. Analysis of the results shown in this table shows that the value of the RMAE criterion decreases with the forecasting horizon. It begins to grow with the horizon from Week 4 onwards. It increases with the forecasting horizon for the other criteria. This is explained by the fact that the series does not possess long-term dynamics and does possess a short-term component. Indeed, the movements observed appear to be the result of transitory exogenous shocks affecting the Paris market; the consequences of the shocks are therefore transitory, with a return of the price to its fundamental value. We therefore consider that as short term market returns can be forecast, it is not possible a priori to develop profitable market strategies. The market is not weak-form efficient and prices do not reflect all the information available.

\section{Conclusion}

We have examined the efficiency of the Paris stock market by examining the behaviour of the CAC40 share price index using a non-parametric approach. We have shown that the method resulted in better forecasts than those given by a simple random operation model. A first technical result shows the advantage of use of the kernel method. It makes it possible to confirm and sometimes improve the results obtained using the traditional methods for the analysis of time series. It is therefore a serious alternative to the non-linearity hypothesis. However, it nonetheless seems necessary to deepen these results to test the efficiency of the French market using not only CAC40 series but also other series such as SBF 250. Finally, we consider that the nonparametric approach is becoming a powerful tool for the study of series of financial figures. Although the structure of the non-linear process is not known, we can now use non-parametric modelling to attempt to reduce our ignorance and decrease the proportion of chance. We can also improve our conception of the random by quantitative evaluation of the degree of randomness of the dynamic systems used to model series of financial figures. 


\section{References}

Auestad, B. \& Tjosthem, D. (1990), Identification of nonlinear time series: first order characterization and order determination, Biometrika, 77, 669-687.

Box, G \& Pierce, D. (1970), Distribution of residual autocorrelation in autoregressive integrated moving average time series models, J. Ann. Statistic., 6, 461-464.

Breitung, J. (2002), Nonparametric tests for unit roots and cointegration, Journal of Econometrics, 108, 343-364.

Breusch, T.S \& Agan, A.R. (1979), A Simple Test for Heteroscedasticity and Random Coefficient Variation, Econometrica, 47, 1287-1294.

Brock, W.A, Dechert, W.D. \& Scheinkman, J.A. (1987), A test for independence based on the correlation dimension, Working Paper, University of Wisconsin.

Brockmann, M. (1993), Locally adaptive bandwidth choice for kernel regression estimators, J. Amer. Statist. Assoc., 88, 1302-1309.

Cheng, B. \& Tong, H. (1992), On consistent nonparametric order determination and chaos, Journal of The Royal Statistical Society, Series B, 54, 427-449.

Cheung, Y.W. (1993), Tests for Fractional Integrationn: A Monte Carlo Investigation, Journal of Time Series Analysis, 14, 331-345.

Cobbaut, R. (1997), Théorie financière, Economica, Paris.

Chiu, S.T. (1989), Bandwidth selection for kernel estimates with correlated noise, Statist. Probab. Lett., 8, 347-354.

Dickey, D.A \& Fuller, W.A. (1981), The likelihood ratio statistics for autoregressive time series with a unit root”, Journal of the American Statistical Association, 74, 427-431.

Doukhan, P. (1994), Mixing: properties and examples, Springer, Berlin.

Engle, R.F. (1982), Autoregressive conditional heteroscedasticity with estimates of the variance of United Kingdom inflation, Econometrica, 50, 987-1007.

Fama, E.F. (1991), Efficient capital markets, Journal of Finance, XLVI, 1575-1617.

Fontaine, P. (1990), Peut-on prédire l'évolution des marchés d'actions à partir des cours et des dividendes passés?, Journal de la Société Statistique de Paris, 131, 16-36.

Geweke, J \& Porter-Hudak, S. (1983), The estimation and application of long memory time series models, Journal of Time Series Analysis, 4, 221-238.

Gillet, P. (1999), L’efficience des marchés financiers, Economica, Paris.

Gouriéroux, C. (1992), Modèles ARCH et applications financières, Economica, Paris.

Härdle, W \& Chen, R. (1996), Nonparametric Time Series Analysis, a selective review with examples, Proceedings of the $50^{\text {th }}$ session of the ISI, Peking. 
Härdle, W \& Yang, L. (1996), Nonparametric autoregression with Multiplicative Volatility and additive Mean, Discussion paper 96-62, SFB 373, HumboldtUniversität zu Berlin.

Härdle, W, Lütkepohl, H \& Chen, R. (1996), A review of nonparametric time series analysis, Discussion Paper 96-48, SFB 373, Humboldt-Universität zu Berlin.

Härdle, W, Tsybakov, A.B. \& Yang, L. (1998), Nonparametric vector autoregression, Journal of Statistical Planning and Inference, 68, 221-245.

Härdle, W. (1990), Applied nonparametric regression, Cambridge University Press, Cambridge.

Jacquillat, B \& Solnik, B. (1997), Marchés financiers: Gestion de portefeuille et des risques, Dunod, Paris.

Jarque, C.M \& Bera, A.K. (1980), Efficient Tests for Normality, homoscedasticity and serial independence of regression residuals, Economics Letters, 6, 255-259.

Masry, E \& Tjostheim, D. (1995), Nonparametric estimation and identification of nonlinear ARCH time series: strong convergence and asymptotic normality, Econometric Theory, 11, 258-289.

Mignon, V. (1998), Marchés financiers et modélisation des rentabilités boursières, Economica, Paris.

Mizrach, B. (1996), A simple nonparametric test for independence, Working Paper n¹995-23, Rutgers University, Department of Economics.

Phillips, P.C.B \& Perron, P. (1988), Testing for a unit root in time series regression, Biometrika, 75, 335-346.

Robinson, P.M. (1983), Nonparametric estimators for time series, Journal of Time Series Analysis, 4, 185-207.

Silverman, B.W. (1986), Density estimation for Statistics and data analysis, Chapman \& Hall, London.

Tjostheim, D. \& Auestad, B. (1994), Nonparametric identification of nonlinear time series: selecting significant lags, Journal of American Statistical Association, 89, 1410-1419.

Tschernig, R \& Yang, L. (2000), Nonparametric lag selection for time series, Journal of Time Series Analysis, 21, 457-487.

Vieu, P. (1995), Order choice in nonlinear autoregressive models, Statistics, 27, 307328.

White, H. (1980), A hetetoscedasticity-consistent covariance estimator and a direct test for heteroscedasticity, Econometrica, 48, 817-838.

Yang, L \& Tschernig, R. (1999), Multivariate bandwidth selection for local linear regression, Journal of the Royal Statistical Society, Series B, 61, 793-815. 


\section{Appendix}

\begin{tabular}{|c|c|c|}
\hline \multirow{2}{*}{ Model } & $\begin{array}{c}\text { DF Statistic on the } \\
\text { CAC40 Series }\end{array}$ & $\begin{array}{c}\text { DF Statistic on the } \\
\text { CAC40 Returns Series }\end{array}$ \\
\hline \multirow{2}{*}{ None } & 1.908 & -24.814 \\
& $(-1.939)$ & $(-1.939)$ \\
\hline \multirow{2}{*}{ Intercept } & 0.785 & -24.863 \\
$(-2.863)$ & $-2.863)$ \\
\hline \multirow{2}{*}{ Trend and Intercept } & -1.024 & $(-3.413)$ \\
\hline
\end{tabular}

Table 1 - Augmented Dickey-Fuller Unit Root Test

\begin{tabular}{|c|c|c|c|c|}
\hline \multirow[b]{2}{*}{ Model } & \multicolumn{2}{|c|}{ Phillips-Perron Test } & \multicolumn{2}{|c|}{ Breitung's Nonparametric Test } \\
\hline & CAC40 Series & $\begin{array}{c}\text { CAC40 } \\
\text { Returns Series }\end{array}$ & CAC40 Series & $\begin{array}{c}\text { CAC40 } \\
\text { Returns Series }\end{array}$ \\
\hline None & $\begin{array}{c}2.134 \\
(-1.939) \\
\end{array}$ & $\begin{array}{l}-53.383 \\
(-1.939) \\
\end{array}$ & \multirow{2}{*}{$\begin{array}{c}\text { Stationarity: } \\
0.0104 \\
(0.063)\end{array}$} & \multirow{2}{*}{$\begin{array}{c}\text { Stationarity: } \\
0.0104 \\
(0.00006)\end{array}$} \\
\hline Intercept & $\begin{array}{c}1.032 \\
(-2.863)\end{array}$ & $\begin{array}{l}-53.414 \\
(-2.863)\end{array}$ & & \\
\hline $\begin{array}{l}\text { Trend and } \\
\text { Intercept }\end{array}$ & $\begin{array}{c}-0.836 \\
(-3.413)\end{array}$ & $\begin{array}{c}-53.431 \\
(-3.4139)\end{array}$ & $\begin{array}{c}\text { Trend } \\
\text { Stationarity: } \\
0.0035 \\
(0.012)\end{array}$ & $\begin{array}{c}\text { Trend } \\
\text { Stationarity: } \\
0.00355 \\
(0.00002)\end{array}$ \\
\hline
\end{tabular}

Table 2 - Nonparametric Phillips-Perron and Breitung's Unit Root Tests

\begin{tabular}{cccc}
\hline Series & Skewness & Kurtosis & Jarque-Bera stat. \\
\hline CAC40 returns & -0.447 & 9.210 & 5017.611 \\
\hline
\end{tabular}

Table 3 - Jarque-Bera Normality Test for the CAC40 Returns Series

\begin{tabular}{ccc}
\hline Series & Breusch-Pagan Statistic & White statistic \\
\hline CAC40 Returns & 764.25 & 660.415 \\
\hline
\end{tabular}

Table 4 - Heteroscedasticity Tests 


\begin{tabular}{|c|c|}
\hline$m$ & Mizrach Statistic \\
\hline 1 & 3.495 \\
\hline 2 & 4.072 \\
\hline 3 & 4.951 \\
\hline 4 & 3.537 \\
\hline 5 & 2.969 \\
\hline 6 & 2.566 \\
\hline 7 & 2.381 \\
\hline 8 & 2.51 \\
\hline 9 & 2.648 \\
\hline 10 & 2.326 \\
\hline
\end{tabular}

Table 5 - Nonparametric Mizrach Test for the CAC40 Returns Series

\begin{tabular}{|c|c|c|c|c|}
\hline \multirow{2}{*}{$m$} & \multicolumn{4}{|c|}{$\varepsilon / \sigma$} \\
\cline { 2 - 5 } & 0.5 & 1 & 1.5 & 2 \\
\hline 2 & 3.838 & 3.423 & 3.241 & 3.479 \\
\hline 3 & 4.267 & 4.673 & 4.616 & 4.586 \\
\hline 4 & 6.559 & 5.246 & 5.792 & 5.757 \\
\hline 5 & 8.509 & 7.79 & 7.566 & 7.154 \\
\hline
\end{tabular}

Table 6 - Nonparametric BDS Test for the CAC40 Returns Series

\begin{tabular}{|c|c|c|c|c|c|}
\hline \multirow{2}{*}{ Bandwidth } & \multicolumn{5}{|c|}{ Ordinates } \\
\cline { 2 - 6 } & $n^{0.45}$ & $n^{0.5}$ & $n^{0.55}$ & $n^{0.6}$ & $n^{0.65}$ \\
\hline \multirow{2}{*}{ GPH } & 0.088 & 0.089 & 0.119 & 0.059 & 0.042 \\
& $(0.717)$ & $(0.914)$ & $(1.539)$ & $(0.964)$ & $(0.850)$ \\
\hline \multirow{2}{*}{ Rectangular. } & 0.041 & 0.016 & 0.049 & 0.038 & 0.039 \\
& $(0.305)$ & $(0.155)$ & $(0.576)$ & $(0.557)$ & $(0.716)$ \\
\hline \multirow{2}{*}{ Bartlett } & 0.094 & 0.042 & 0.049 & 0.051 & 0.046 \\
& $(1.21)$ & $(0.687)$ & $(0.576)$ & $(1.299)$ & $(1.454)$ \\
\hline \multirow{2}{*}{ Daniell } & 0.095 & 0.042 & 0.068 & 0.051 & 0.046 \\
& $(0.993)$ & $(0.561)$ & $(1.385)$ & $(1.07)$ & $(1.194)$ \\
\hline \multirow{2}{*}{ Tukey } & 0.078 & 0.035 & 0.063 & 0.047 & 0.043 \\
& $(0.92)$ & $(0.517)$ & $(1.178)$ & $(1.112)$ & $(1.269)$ \\
\hline \multirow{2}{*}{ Parzen } & 0.092 & 0.042 & 0.069 & 0.05 & 0.045 \\
& $(1.313)$ & $(0.760)$ & $(1.554)$ & $(1.413)$ & $(1.597)$ \\
\hline \multirow{2}{*}{ B-priest } & 0.062 & 0.027 & 0.057 & 0.043 & 0.041 \\
& $(0.59)$ & $(0.325)$ & $(0.867)$ & $(0.825)$ & $(0.984)$ \\
\hline
\end{tabular}

Table 7 - Semiparametric Estimates of the ARFIMA Models for the CAC40 Returns Series 


\begin{tabular}{cccc}
\hline Model & Selected lags & CAFPE $_{\text {opt }}$ & The bandwidth $h$ \\
\hline$N A R$ & $2,3,7$ & 0.901 & 0.787 \\
\hline
\end{tabular}

Table 8 - Nonparametric Selection of the NAR Model

\begin{tabular}{cccc}
\hline Series & Skewness & Kurtosis & Jarque-Bera stat. \\
\hline CAC40 returns & -0.447 & 9.210 & 5017.611 \\
\hline
\end{tabular}

Table 9 - Jarque-Bera Normality Test for the Residuals of NAR Model

\begin{tabular}{|c|c|}
\hline$m$ & Mizrach statistic \\
\hline 1 & 1.416 \\
\hline 2 & -2.285 \\
\hline 3 & -2.209 \\
\hline 4 & -2.497 \\
\hline 5 & -1.101 \\
\hline
\end{tabular}

Table 10 - Nonparametric Mizrach Test for the Residuals of NAR model

\begin{tabular}{cccc}
\hline Model & Selected Lags & CAFPE $_{\text {opt }}$ & The Bandwidth $h$ \\
\hline $\begin{array}{c}\text { Conditional } \\
\text { Volatility }\end{array}$ & $4,5,7$ & 0.897 & 0.698 \\
\hline
\end{tabular}

Table 11 - Nonparametric Estimation of the Conditional Volatility

\begin{tabular}{|c|c|}
\hline$m$ & Mizrach statistic \\
\hline 1 & 0.216 \\
\hline 2 & -1.085 \\
\hline 3 & -1.109 \\
\hline 4 & -0.597 \\
\hline 5 & -0.201 \\
\hline
\end{tabular}

Table 12 - Nonparametric Mizrach Test for the Standardised Residuals of NAR-ARCH Model

\begin{tabular}{|c|c|c|c|}
\hline Steps Ahead & Criterion & $\begin{array}{c}\text { Nonparametric } \\
\text { NAR-ARCH }\end{array}$ & Random Walk \\
\hline \multirow{3}{*}{$1^{\text {st }}$ week } & MRAE & $370.19 \%$ & $370.24 \%$ \\
\cline { 2 - 4 } & QME & $0.7 \%$ & $0.74 \%$ \\
\cline { 2 - 4 } & MAE & $0.79 \%$ & $0.82 \%$ \\
\cline { 2 - 4 } & RSE & $0.84 \%$ & $0.86 \%$ \\
\hline \multirow{3}{*}{$2^{\text {nd }}$ week } & MRAE & $301.65 \%$ & $302.33 \%$ \\
\cline { 2 - 4 } & QME & $0.83 \%$ & $0.84 \%$ \\
\cline { 2 - 4 } & MAE & $0.78 \%$ & $0.83 \%$ \\
\cline { 2 - 4 } & RSE & $0.86 \%$ & $0.88 \%$ \\
\hline
\end{tabular}




\begin{tabular}{|c|c|c|c|}
\hline \multirow{3}{*}{$3^{\text {rd }}$ week } & MRAE & $228.12 \%$ & $228.25 \%$ \\
\cline { 2 - 4 } & QME & $0.11 \%$ & $0.16 \%$ \\
\cline { 2 - 4 } & MAE & $0.93 \%$ & $0.94 \%$ \\
\cline { 2 - 4 } & RSE & $1.23 \%$ & $1.27 \%$ \\
\hline \multirow{3}{*}{$4^{\text {th }}$ week } & MRAE & $330.99 \%$ & $330.99 \%$ \\
\cline { 2 - 4 } & QME & $2.27 \%$ & $2.29 \%$ \\
\cline { 2 - 4 } & MAE & $1.09 \%$ & $1.11 \%$ \\
\cline { 2 - 4 } & RSE & $1.61 \%$ & $1.61 \%$ \\
\hline
\end{tabular}

Table 13 - Comparison of Forecasts

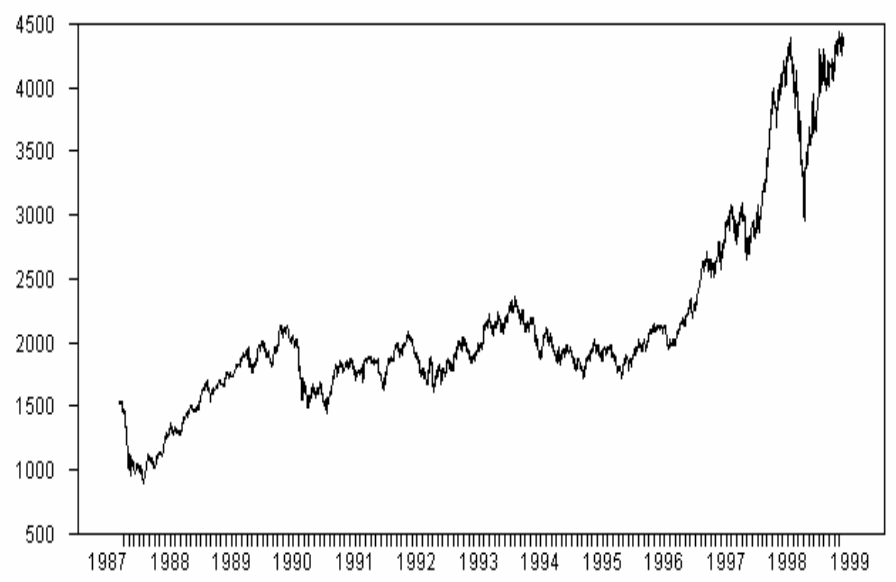

Figure 1 - The Stock Exchange CAC40 series

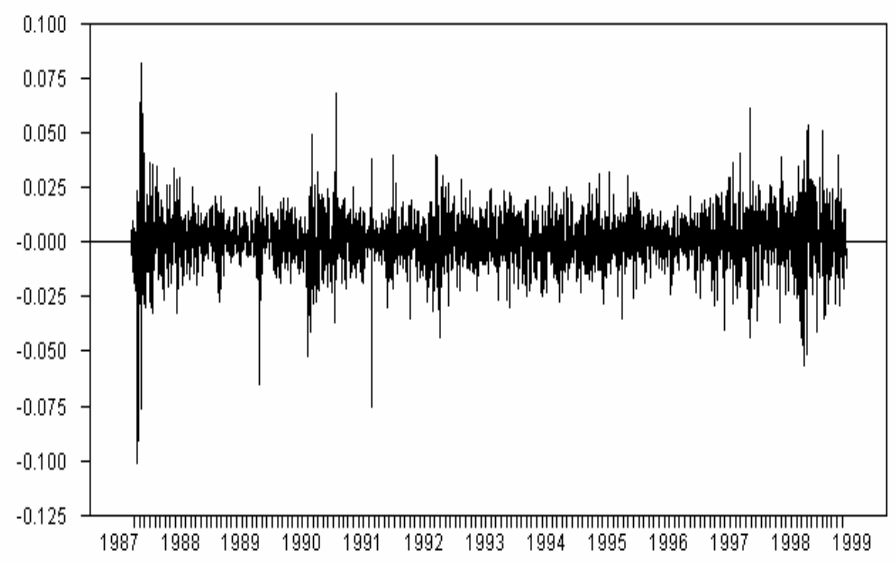

Figure 2 - The Stock Exchange CAC40 Returns Series 
Kennel estimate of the density $f(y)$ of $Y=D C A C$

with bandwidth $\mathrm{h}=\mathrm{c} \cdot \mathrm{n}^{\wedge}(-1 / 5)$, where $\mathrm{c}=1$, compared with the corresponding normal density (dashed curve)

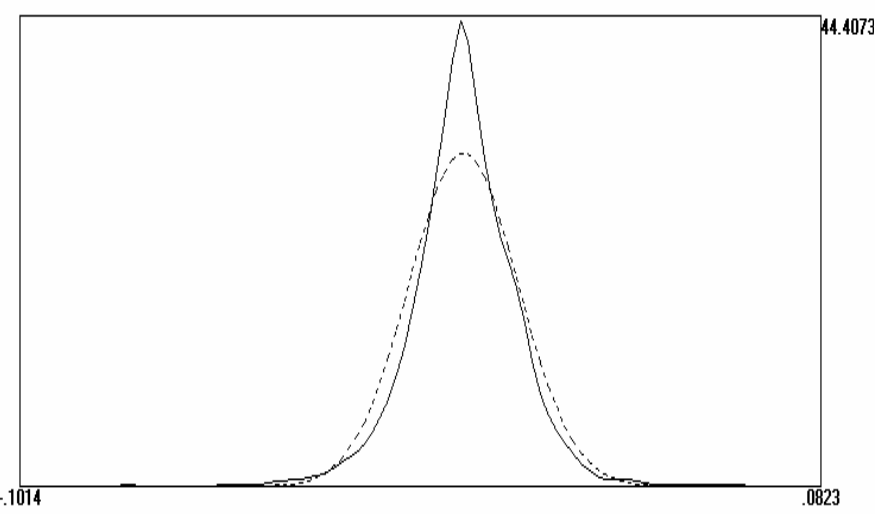

Figure 3 - The Nonparametric Kernel Density Estimator

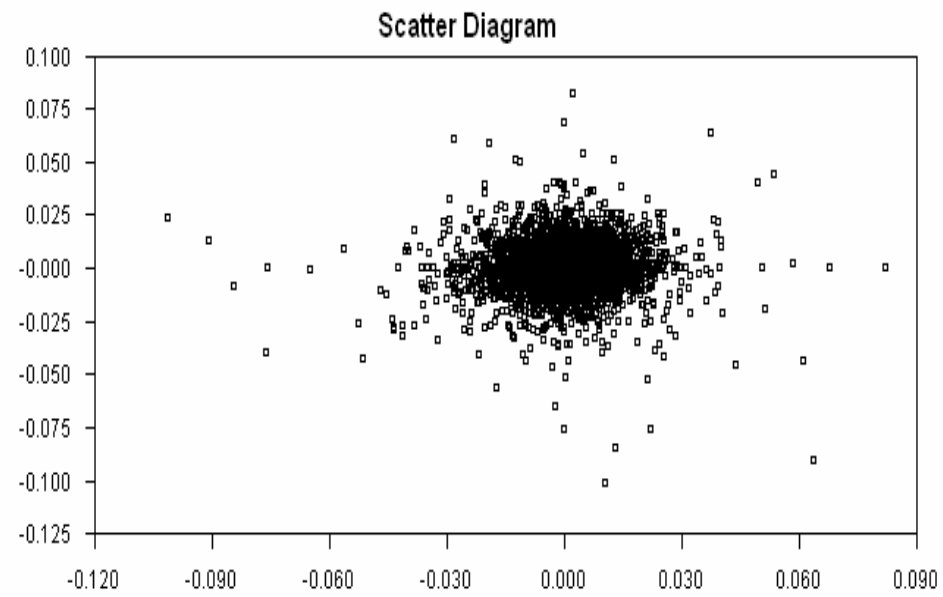

Figure 4 - Scatter Diagram Attractor of Returns Series

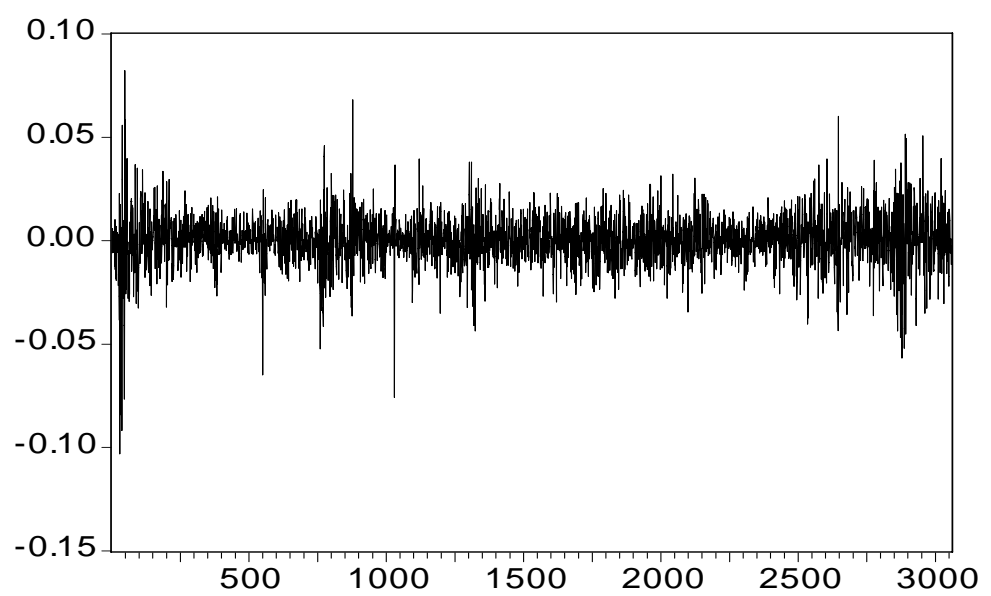

Figure 5 - Residuals of the Nonparametric NAR Model using the Lags 2 and 7 


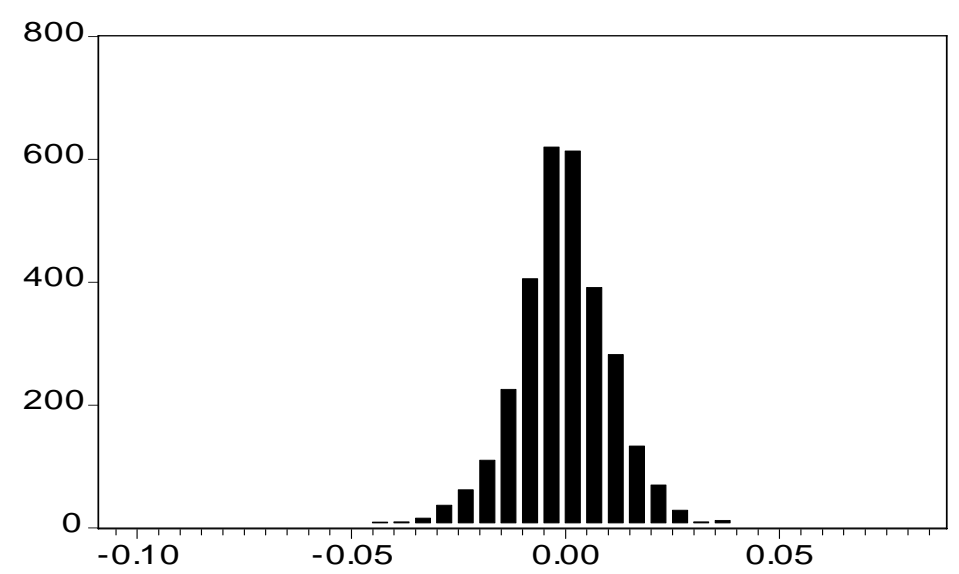

Figure 6 - Histogram of the Distribution of Residuals

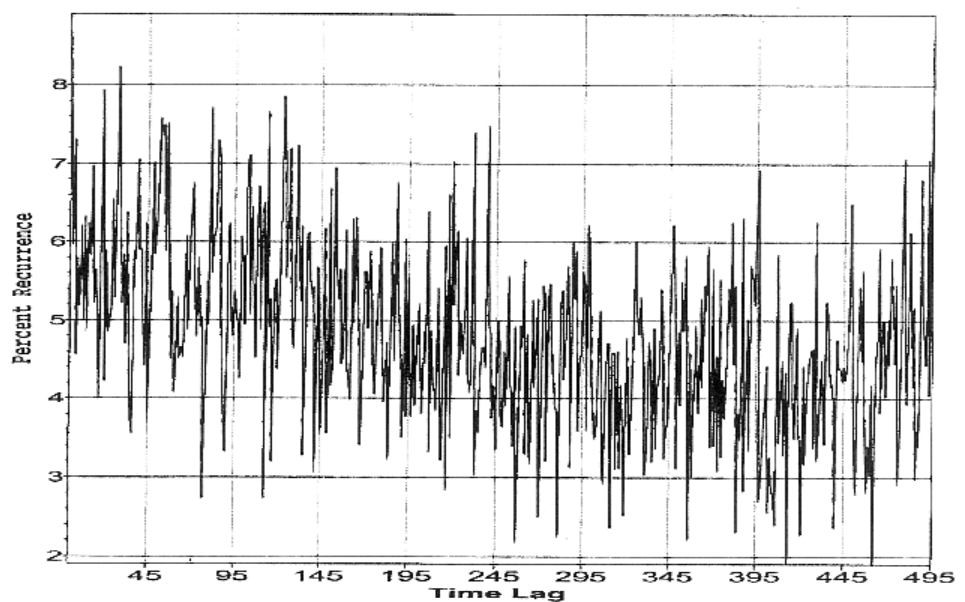

Figure 7 - Recurrence Histogram of the Residuals for the Nonparametric NAR Model 



\section{WORKING PAPERS DE L'AFC}

\section{Année 2006}

WP2006-1 Olivier DARNÉ, Claude DIEBOLT

"Cliometrics of Academic Careers and the Impact of Infrequent Large Shocks in Germany before 1945"

WP2006-2 Claude DIEBOLT, Catherine KYRTSOU

"Non-Linear Perspectives for Population and Output Dynamics: New Evidence for Cliometrics"

WP2006-3 Claude DIEBOLT, Karine PELLIER

"L'intérêt des systèmes de gestion de bases de données relationnels en cliométrie"

WP2006-4 Claude DIEBOLT, Jean-Pascal GUIRONNET

"The Dynamics of Education Returns"

WP2006-5 Claude DIEBOLT, Jean-Pascal GUIRONNET

"Vers une théorie économique de la suréducation ?"

WP2006-6 Claude DIEBOLT, Mishra TAPAS

"Cliometrics of the Abiding Nexus between Demographic Components and Economic Development"

WP2006-7 Magali JAOUL

"Cliométrie de l'engorgement en France.

Evaluation théorique et empirique"

WP2006-8 Claude DIEBOLT, Antoine PARENT

"Were the Anomalies in the Sterling-Franc Exchange Rate Regulation during the Mid-19th Century?"

WP2006-9 Claude DIEBOLT

"Progrès technique et cycles économiques dans la pensée allemande de l'entre-deux-guerres : I'apport d'Emil Lederer"

WP2006-10 Claude DIEBOLT

"Croissance et éducation"

WP2006-11 Mohamed CHIKHI, Claude DIEBOLT

"Nonparametric Analysis of Financial Time Series by the Kernel Methodology"

WP2006-12 Claude DIEBOLT, Antoine PARENT

"A Note on Juglar, Bonnet and the Intuition of the Interest

Parity Relation"

La liste complète des Working Papers est disponible sur le site www.cliometrie.org 